AIP $\mid \begin{aligned} & \text { Joumalof } \\ & \text { Applied Physics }\end{aligned}$

\title{
Precessional dynamics in microarrays of nanomagnets
}

V. V. Kruglyak, A. Barman, R. J. Hicken, J. R. Childress, and J. A. Katine

Citation: J. Appl. Phys. 97, 10A706 (2005); doi: 10.1063/1.1849057

View online: http://dx.doi.org/10.1063/1.1849057

View Table of Contents: http://jap.aip.org/resource/1/JAPIAU/v97/i10

Published by the American Institute of Physics.

\section{Additional information on J. Appl. Phys.}

Journal Homepage: http://jap.aip.org/

Journal Information: http://jap.aip.org/about/about_the_journal

Top downloads: http://jap.aip.org/features/most_downloaded

Information for Authors: http://jap.aip.org/authors

\section{ADVERTISEMENT}

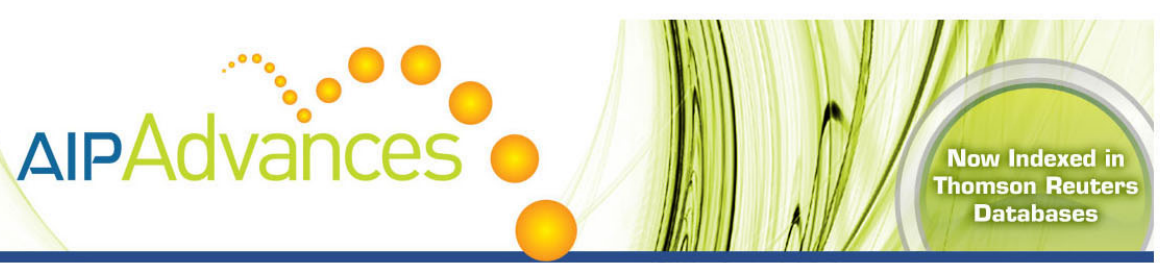

\section{Explore AIP's open access journal: Rapid publication \\ - Article-level metrics \\ - Post-publication rating and commenting}




\title{
Precessional dynamics in microarrays of nanomagnets
}

\author{
V. V. Kruglyak, A. Barman, and R. J. Hicken ${ }^{\text {a) }}$ \\ School of Physics, University of Exeter, Stocker Road, Exeter, EX4 4QL, UK \\ J. R. Childress and J. A. Katine \\ Hitachi Global Storage Technologies, San Jose Research Center, 650 Harry Road, San Jose, \\ California 95120
}

(Presented on 11 November 2004; published online 28 April 2005)

\begin{abstract}
Time resolved scanning Kerr microscopy has been used to study the response of square $\mathrm{Ni}_{88} \mathrm{Fe}_{12} / \mathrm{Co}_{80} \mathrm{Fe}_{20}$ bilayer elements to a pulsed magnetic field. Measurements were performed upon a square element of $6000 \mathrm{~nm}$ size and upon 64,120, 220, 425, and $630 \mathrm{~nm}$ square elements that formed square arrays of about $4000 \mathrm{~nm}$ total size. While the frequency of precession of the magnetization of the $6000 \mathrm{~nm}$ element could be described with a macrospin model, the frequencies observed in the arrays of submicron size elements differed from the macrospin prediction. This observation may be understood in terms of the increasing nonuniformity of the demagnetizing field as the element aspect ratio is decreased. (C) 2005 American Institute of Physics.
\end{abstract}

[DOI: 10.1063/1.1849057]

Patterned thin film magnetic materials are one of the main subjects of investigation within modern magnetism. The dynamical properties of these materials have been studied experimentally by Brillouin light scattering, ${ }^{1}$ time resolved scanning Kerr microscopy ${ }^{2}$ (TRSKM), ferromagnetic resonance, ${ }^{3,4}$ and pulsed inductive microwave magnetometry. ${ }^{5}$ However, current experimental methods do not yet provide sufficient spatial resolution for the dynamical properties of a single nanoscale element to be studied directly. Consequently the magnetic properties of the element must be deduced from measurements made on the entire array. ${ }^{1,3,4}$ In this article, measurements of the precessional dynamics in $\mathrm{Ni}_{88} \mathrm{Fe}_{12}(27 \AA) / \mathrm{Co}_{80} \mathrm{Fe}_{20}(10 \AA)$ single microelement and nanoelement arrays are presented, using the TRSKM as a submicron probe of the magnetization dynamics at the center of a sample.

A square element of $6000 \mathrm{~nm}$ size and square arrays of about $4000 \mathrm{~nm}$ total size with 64, 120, 220, 425, and $630 \mathrm{~nm}$ elements were patterned using electron beam lithography. Scanning electron microscope images showed that the 220 , 425,630 , and $6000 \mathrm{~nm}$ elements have a perfect square shape, while the 64 and $120 \mathrm{~nm}$ elements came out slightly rounded. The nominal composition of the elements was $\mathrm{Si} / \mathrm{Ta}(50 \AA) / \mathrm{Co}_{80} \mathrm{Fe}_{20}(10 \AA) / \mathrm{Ni}_{88} \mathrm{Fe}_{12}(27 \AA) / \mathrm{Ta}(100 \AA)$. A comparison of the results of static magnetometry measurements made upon sheet samples of different thickness codeposited with the elements showed that $12.1 \AA$ of $\mathrm{Ni}_{88} \mathrm{Fe}_{12}$ was lost due to interdiffusion with Ta, leaving a $\mathrm{Ni}_{88} \mathrm{Fe}_{12}$ layer with thickness of $14.9 \AA$. The compositions of the $\mathrm{Ni}_{88} \mathrm{Fe}_{12}$ and $\mathrm{Co}_{80} \mathrm{Fe}_{20}$ alloys were chosen so that the magnetostriction of these two layers would compensate each other, and the bilayer as a whole would have vanishing magnetostriction. ${ }^{6}$ The $\mathrm{Co}_{80} \mathrm{Fe}_{20}$ alloy composition gave a stable fcc structure. The direction of the easy axis was set in the samples by the field annealing. The magnetizations of the

${ }^{a)}$ Electronic mail: R.J.Hicken@ex.ac.uk
$\mathrm{Co}_{80} \mathrm{Fe}_{20}$ and $\mathrm{Ni}_{88} \mathrm{Fe}_{12}$ were deduced to be equal to 1445 and $585 \mathrm{emu} / \mathrm{cm}^{3}$, respectively. Since the two layers were strongly coupled by the interlayer exchange interaction, we can treat them as a single layer with thickness equal to the sum of their thicknesses (24.9 $\AA$ ), and with magnetization given by an arithmetical average of their saturation magnetizations $\left(930 \mathrm{emu} / \mathrm{cm}^{3}\right)$. The uniaxial and average surface anisotropy constants $K_{2}$ and $K_{s}$ were found to be equal to $5120 \mathrm{erg} / \mathrm{cm}^{3}$ and $0.11 \mathrm{erg} / \mathrm{cm}^{2}$, respectively.

The elements were formed in between the tracks of a $\mathrm{Au}$ transmission line structure with a $30 \mu \mathrm{m}$ track width and separation so as to experience an out-of-plane pulsed magnetic field. The measurements were performed in the TRSKM configuration at a wavelength of $790 \mathrm{~nm}$, as described in detail elsewhere. ${ }^{7}$ In this configuration, the measured signal was proportional to the polar Kerr rotation, and hence, to the out-of-plane component of the magnetization. In measurements made upon the element arrays, several elements were typically in the area probed by the microscope. Hence, the measurement was sensitive only to the average response of the elements, falling under the probe spot. A photoconductive switch made from $\mathrm{Au}$ on a GaAs substrate was connected to the transmission line structure, and used for generation of the pulsed magnetic field. The optically gated pulsed field had a rise time of about 40 ps and a decay time of about 2 ns. A static field $\mathbf{H}$ was applied in the plane of the sample and its strength and orientation were varied during the experiment. Each value of the static field was approached from a much greater value, typically about $1-2 \mathrm{kOe}$, that was sufficient to cause saturation.

Two types of dynamic Kerr measurement were performed with the probe spot focused at the center of the $6000 \mathrm{~nm}$ element. First, the static field strength $\mathrm{H}$ was varied with the field vector $\mathbf{H}$ parallel and perpendicular to the tracks of the transmission line structure, i.e., parallel to the expected directions of the easy (EA) and hard (HA) anisotropy axes of the element, respectively. Figure 1 shows typi- 

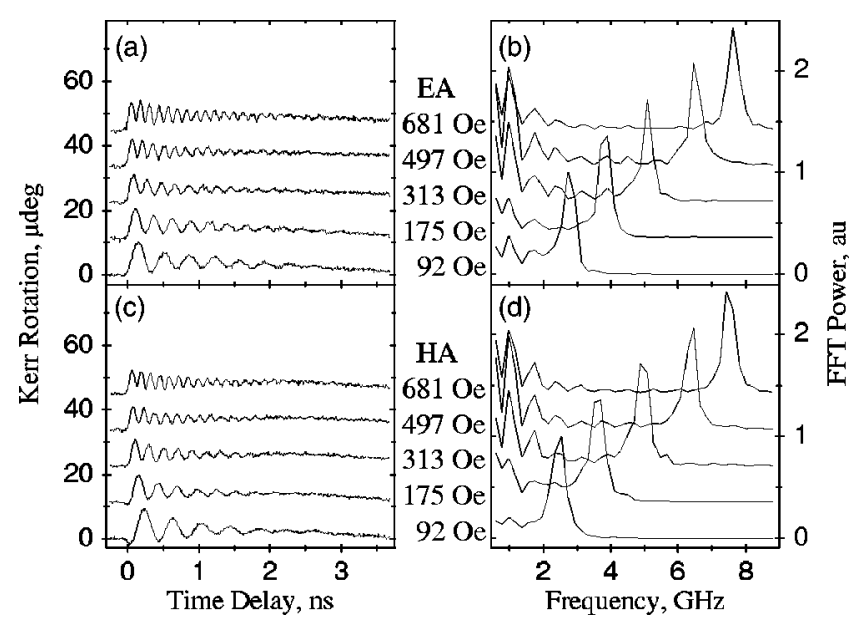

FIG. 1. Typical time resolved Kerr rotation signals measured from the $6000 \mathrm{~nm}$ element are presented in panels (a) and (b) for different values of the bias field applied parallel to the EA and HA directions, respectively. The Fourier spectra of the corresponding signals in panels (a) and (b) are presented in panels (c) and (d), respectively.

cal time dependent Kerr signals and their fast Fourier transform spectra. Since the oscillations decayed well within the duration of the scan, the Fourier transforms were performed using a rectangular window function. As well as the field dependent peaks at higher frequencies, one can also see lower frequency peaks, the positions of which do not depend upon the bias field strength. The latter peaks originate from the pulsed field profile and can be clearly distinguished from the sample response. However, an accurate determination of frequency seems infeasible for frequencies less than approximately $2 \mathrm{GHz}$. Kerr images of the dynamic magnetization distribution acquired at different time delays between the pump and the probe (not shown) did not reveal any spatial nonuniformity. Hence, the only observed mode of magnetization precession was identified as the uniform mode. In a second type of measurement (not shown), the direction of the bias field was varied, while its magnitude was kept constant at a value of 240 Oe, allowing the symmetry and magnitude of the in-plane anisotropy to be more directly determined.

The data from both sets of measurements were fitted to a macrospin model for a continuous magnetic film (see Fig. 2). ${ }^{7}$ In the fitting, the magnetization was assumed to be always parallel to the bias magnetic field. The $g$-factor and the saturation magnetization were fixed at values of 2.1 and $930 \mathrm{emu} / \mathrm{cm}^{3}$, respectively. The fitted uniaxial and surface anisotropy parameters had values of $4140 \pm 330 \mathrm{erg} / \mathrm{cm}^{3}$ and $0.156 \pm 0.022 \mathrm{erg} / \mathrm{cm}^{2}$. The sample EA was canted by about $10^{\circ}$ from the expected direction parallel to the tracks of the transmission line structure. However, throughout this article we mean by the EA and HA directions their expected directions, parallel to which the bias field was actually applied during the measurements.

Typical Kerr signals and their Fourier spectra obtained from arrays with 630, 425, 220, 120, and $64 \mathrm{~nm}$ size elements are presented in Fig. 3. Figure 4 presents the frequencies of the observed modes together with curves obtained by fitting the frequencies of the $6 \mu \mathrm{m}$ element to the macrospin model equations. The deviation from the latter curves is rela-

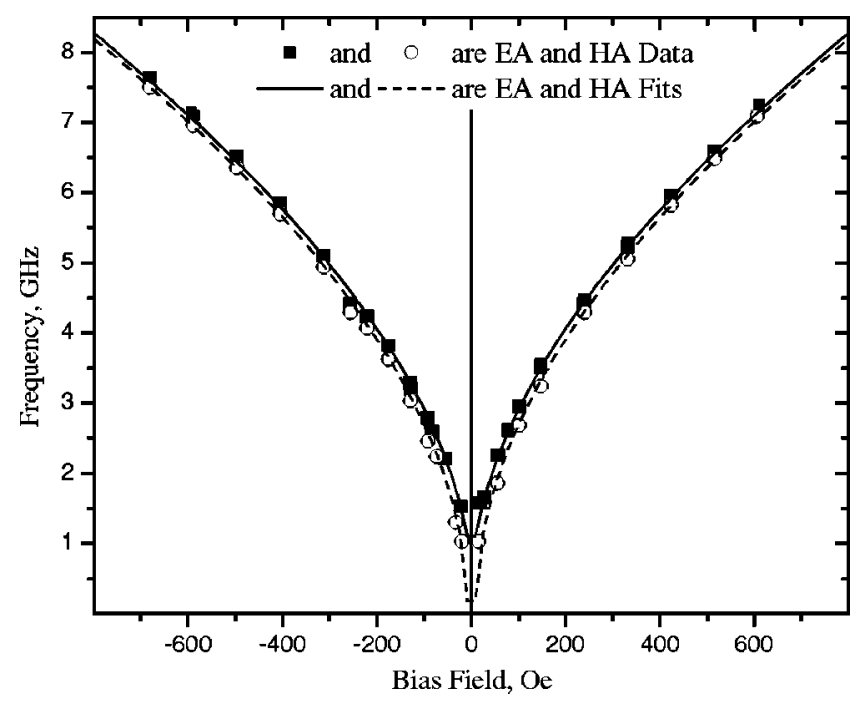

FIG. 2. The dependence of the uniform mode frequency upon the bias field magnitude is presented for the $6000 \mathrm{~nm}$ element. The symbols are data points and the lines are least-square fits to the macrospin model equations.

tively small for the 425 and $630 \mathrm{~nm}$ element arrays and much greater for the 220, 110, and $64 \mathrm{~nm}$ element arrays. The dependence of the mode frequencies upon the element size is presented in Fig. 5 for the field applied parallel to the EA direction. The dependence is complicated and nonmonotonic. For the 630, 425, and $220 \mathrm{~nm}$ element arrays, the frequency increases as the element size decreases. In the 120 and $64 \mathrm{~nm}$ element arrays, the same trend is observed, but the frequencies are reduced in comparison with those in the 630,425 , and $220 \mathrm{~nm}$ element arrays. The line width of the Fourier peaks was noticeably greater in the 120 and $64 \mathrm{~nm}$ arrays in comparison with the 630 and $425 \mathrm{~nm}$ element arrays.

We begin by comparing the observed frequencies with those predicted by a macrospin model modified to account for the demagnetizing field of the nanoscale elements. Figure 5 presents the measured precession frequencies and the frequencies calculated for individual elements with size equal
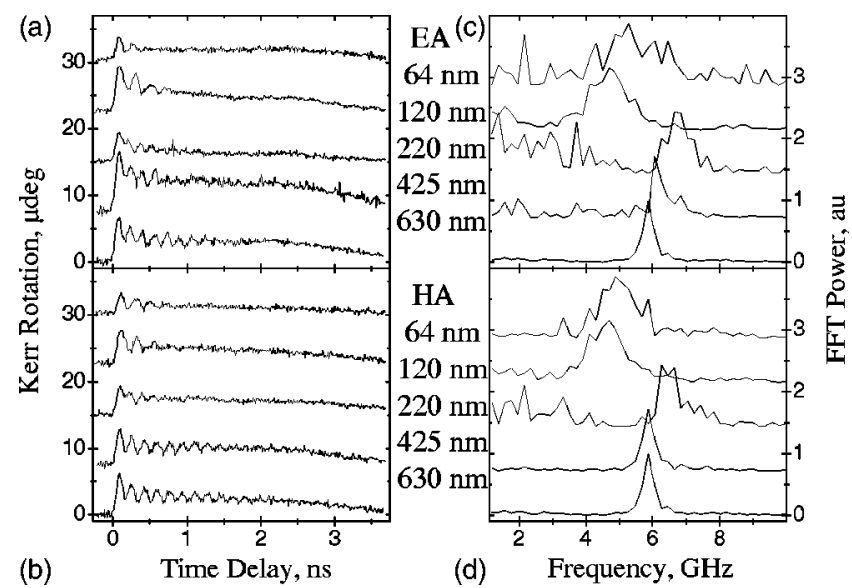

FIG. 3. Typical time resolved Kerr rotation signals obtained from the different element arrays are presented in panels (a) and (b) for the bias field of $405 \mathrm{Oe}$ applied parallel to the EA and HA directions, respectively. The Fourier spectra of the corresponding signals in panels (a) and (b) are presented in panels (c) and (d), respectively. 


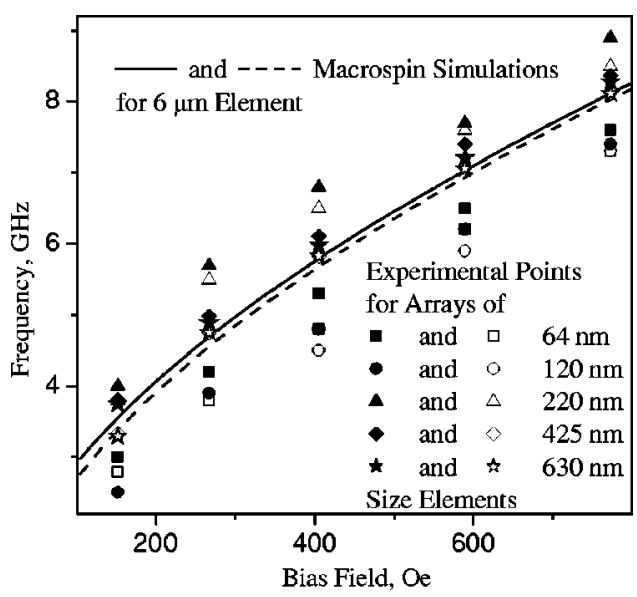

FIG. 4. The mode frequencies obtained from different element arrays are plotted as a function of the bias field magnitude. The lines are the macrospin model fits of the $6000 \mathrm{~nm}$ element frequencies from Fig. 2. The solid symbols and solid line correspond to the bias field parallel to the EA direction. The open symbols and the dashed line correspond to the bias field parallel to the HA direction.

to those in the arrays. The calculations were performed with the object oriented micromagnetic framework (OOMMF) package. ${ }^{8}$ The cell size was set to be equal to the size of the element, which corresponds to a uniform distribution of the magnetization in the element. An out-of-plane pulsed field with $40 \mathrm{ps}$ rise time, 2 ns decay time, and magnitude of 15 Oe was applied to the sample, and the magnetization was recorded after every 5 ps. The OOMMF implements analytical formulas derived in Ref. 9 for the average demagnetizing tensor components of a uniformly magnetized prism. Hence, each calculated frequency corresponds to a solution of the macrospin model corrected to account for the demagnetizing field. Although the experimental data follow the calculated frequencies "on average," the detailed dependence on the element size is not properly reproduced. Hence, no further

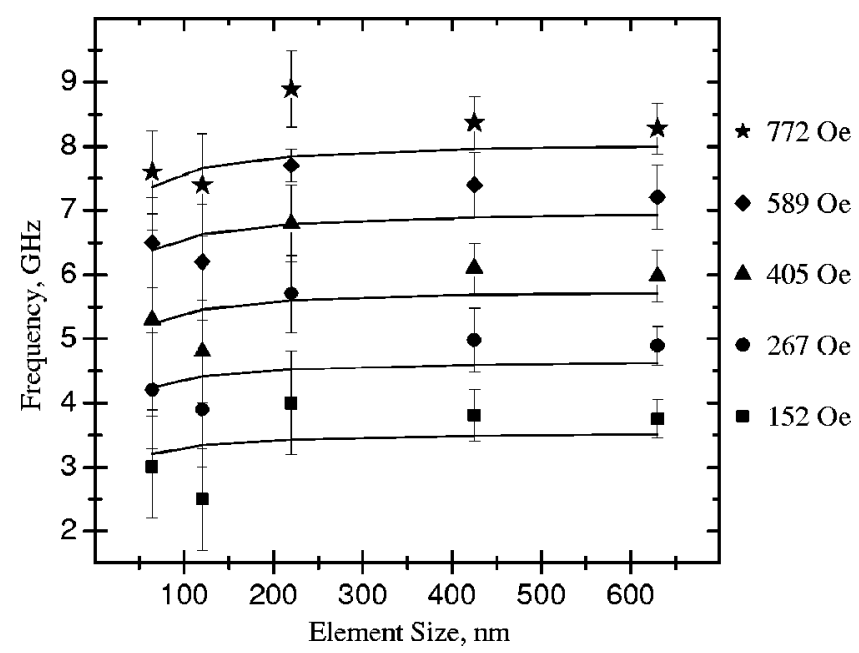

FIG. 5. The extracted mode frequencies are plotted as a function of the element size for different values of the bias field parallel to the EA direction. The symbols represent data points, and the curves are simulations with OOMMF under assumption that the elements were uniformly magnetized. attempts were made to analyze the frequencies with the macrospin model.

We now briefly consider other possible reasons for the observed discrepancy between the experimental and simulated frequencies. While the macrospin simulations assumed that the elements had a square shape, the 64 and $120 \mathrm{~nm}$ elements were slightly rounded. The demagnetizing fields of a prism and a disk of the same aspect ratio ${ }^{9}$ are different and so the precession frequency is expected to be different. Also, the magnetic properties of smaller elements are more affected by the presence of imperfections. The random nature of the latter will cause different elements in an array to precess at different frequencies, and so the observed frequency peak will be broadened, as was indeed observed in the 64 and $120 \mathrm{~nm}$ element arrays. Another explanation could lie in the collective nature of the observed modes. The field of interaction between different elements in the arrays could shift the frequency from the value predicted by the macrospin model for a single element. ${ }^{4}$ Finally, the observed behavior could be the consequence of nonuniform precession since the frequency of precession may be different in different parts of the element due to the nonuniform distribution of the demagnetizing field. ${ }^{9,10}$ The latter explanation is supported by micromagnetic simulations allowing for the nonuniform magnetization of the elements, details of which will be reported elsewhere.

In summary, the magnetization dynamics of small magnetic elements and element arrays were investigated by means of time resolved optical measurements. While the precession frequency observed for the $6 \mu \mathrm{m}$ element could be described by a macrospin model, the frequencies of the modes observed for arrays of smaller elements showed a more complicated behavior that must be explained using a model that allows for a nonuniform distribution of the magnetization within the elements.

${ }^{1}$ J. Jorzick, S. O. Demokritov, B. Hillebrands, M. Bailleul, C. Fermon, K. Y. Guslienko, A. N. Slavin, D. V. Berkov, and N. L. Gorn, Phys. Rev. Lett. 88, 047204 (2002).

${ }^{2}$ W. K. Hiebert, A. Stankiewicz, and M. R. Freeman, Phys. Rev. Lett. 79, 1134 (1997); Y. Acremann, A. Kashuba, M. Buess, D. Pescia, and C. H. Back, J. Magn. Magn. Mater. 239, 346 (2002); J. P. Park, P. Eames, D. M. Engebretson, J. Berezovsky, and P. A. Crowell, Phys. Rev. Lett. 89, 277201 (2002); A. Barman, V. V. Kruglyak, R. J. Hicken, A. Kundrotaite, and M. Rahman, Appl. Phys. Lett. 82, 3065 (2003); J. Magn. Magn. Mater. 272-276, 2121 (2004); A. Barman, V. V. Kruglyak, R. J. Hicken, J. Scott, A. Kundrotaite, and M. Rahman, J. Appl. Phys. 95, 6998 (2004).

${ }^{3}$ Y. Zhai, J. Shi, X. Y. Zhang, L. Shi, Y. X. Xu, H. B. Haung, Z. H. Lu, and H. R. Zhai, J. Phys.: Condens. Matter 14, 7865 (2002).

${ }^{4}$ G. N. Kakazei, P. E. Wigen, K. Y. Guslienko, V. Novosad, A. N. Slavin, V. O. Golub, N. A. Lesnik, and Y. Otani, Appl. Phys. Lett. 85, 443 (2004).

${ }^{5}$ T. M. Crawford, M. Covington, and G. J. Parker, Phys. Rev. B 67, 024411 (2003).

${ }^{6}$ D. A. Nepela, S. Sarhadi, U.S. Patent No. 5,751,528 (1998).

${ }^{7}$ R. J. Hicken, A. Barman, V. V. Kruglyak, and S. Ladak, J. Phys. D 36, 2183 (2003).

${ }^{8} \mathrm{M}$. Donahue and D. G. Porter, oOMmF USER's GUIDE, Version 1.0, Interagency Report NISTIR 6376, NIST, Gaithersburg, MD, 1999: URL: http:// math.nist.gov/oommf.

${ }^{9}$ A. Aharoni, J. Appl. Phys. 83, 3432 (1998).

${ }^{10}$ M. Pardavi-Horvath, J. Appl. Phys. 95, 6604 (2004). 